'K Thorup, 'V Monk, 'E Gourlay, ${ }^{2 N}$ Aiton. 'Brighton and Sussex Medical School; ${ }^{2}$ Brighton and Sussex University Hospitals NHS Trust, Brighton, UK

Background Fetal Alcohol Syndrome (FAS) comprises a triad of growth impairment, central nervous system dysfunction and characteristic facial features. Diagnosis is complex and often not recognised at an early age. The three facial features: short palpebral fissures, smooth philtrum and thin upper lip, are unique to FAS. Clinical examination is inherently subjective and apart from palpebral fissure length, minimal reference data is available in neonates. Establishing a standardised method and normal range would promote an objective assessment. Earlier diagnosis would enable earlier effective interventions.

Methods Standardised digital facial photographs were taken of normal term Caucasian neonates. Mothers completed anonymous questionnaires about alcohol consumption during pregnancy. Photographs were assessed using Facial Analysis Software to obtain values for palpebral fissure length (PFL) and upper lip circularity (LC). Upper lip thinness and philtrum smoothness were ranked according to 5-point Likert Scale.

Results 29 infants were studied, 17 male: 12 female. Mean gestational age 40.3 weeks (range 37.1-42.3), mean weight 3556g. 23 (79\%) had no prenatal alcohol exposure whilst 6 had minimal exposure (1-2units/week). PFL measurements could be obtained from 21 photographs (72\%) with mean of $15.6 \mathrm{~mm}$ (range 13.7-18.7mm). Upper lip and philtrum values could be determined in 24 (83\%). Mean LC was 57.21 (range 31.4-128.2). Mean rank scores for upper lip and philtrum were both 3 .

Conclusion It has been possible to gain measurements of facial features in just over $3 / 4$ of neonates studied, showing the feasibility of this technique in this age-group. Further results are needed to establish reference ranges.

\section{CONGENITAL DIAPHRAGMATIC HERNIA SURVEILLANCE IN IRELAND}

doi:10.1136/archdischild-2012-302724.0613

1,2J Chukwu, 3,4,5EJ Molloy. 'Clinical Research Unit, National Children's Research Centre; ${ }^{2}$ Molecular \& Cellular Therapeutics, Royal College of Surgeons; ${ }^{3}$ Department of Neonatology, Our Lady's Children's Hospital, Crumlin, Dublin 12; ${ }^{4}$ School of Medicine amd Medical Sciences, University College Dublin; ${ }^{5}$ Neonatology, National Maternity Hospital, Holles Street, Dublin, Ireland

Introduction The incidence of Congenital Diaphragmatic Hernia $(\mathrm{CDH})$ varies from 1 in 2,000 to 15,000 births per annum. Due to the legislation prohibiting termination of any pregnancy in the Republic of Ireland (ROI) we hypothesised that the incidence would be different to international reports. There is no mandatory reporting of $\mathrm{CDH}$ cases in the ROI and Northern Ireland (NI).

Aims To determine the true incidence of $\mathrm{CDH}$ in the ROI and NI. Methods Reporting of $\mathrm{CDH}$ cases to the Irish Paediatric Surveillance Unit (IPSU) based in Dublin started in January 2010. Doctors reported new cases to IPSU using the pro-forma provided. Details of the reported cases were collected using a detailed $\mathrm{CDH}$ questionnaire.

Results 23 cases were reported to the IPSU within the 2 year period consisting of 10 cases in 2010 and 13 in 2011; 8 cases from NI and 15 cases from ROI. The incidence rate in the 2 years using IPSU figures was 0.12 per 1000 live births per annum. The questionnaire was completed in 12 cases. 11/12(92\%) made it to the surgical centres. However the Hospital in Patient System record in one of the three surgical centres indicated that only $6 / 15(40 \%)$ cases managed in that centre in the 2-year period were reported to IPSU

Conclusion It was difficult to determine the true incidence of $\mathrm{CDH}$ using the IPSU data due under-reporting. Increased reporting may be achieved by raising awareness and by the use of capturerecapture mechanism. The establishment of a national congenital anomaly register is also indicated.

\section{THREE SIBLINGS WITH NEONATAL PRESENTATION OF GRISCELLI SYNDROME}

doi:10.1136/archdischild-2012-302724.0614

BR Oandalji. Pediatrics, Ministry of Health, Amman, Jordan

Introduction Griscelli syndrome type 2 is a rare disorder charecterised by pigment dilution (silvery hair), variable immune deficiency, and tendency to develop a life threatening hemophagocytic syndrome. Presentation in neonatal life is even more rare.

Materials and Methods These are three siblings to first cousin parents who presented with Griscelli syndrome in the neonatal life with dessimel outcome.

Results J, A, S, are three siblings, two sisters and one brother born to first cousin parents. All had normal vaginal full term delivery. All presented on day one of life with pallor, silvery hair, and hepatosplenomegaly. Investigations revealed variable degrees of anemia \& thrombocytopenia. BM revealed hemophagocytic syndrome. There was no HLA matched BM donor so they were treated conservatively. They had repeated admissions for infections and received several blood and platalet transfusion. They had stoem courses and died by two months of age.

Conclusion This is probablly the largest series of Griscelli syndrome presenting in neonatal life. BM is the only hope fore this syndrome.

Athorough family history is always helpfull in diagnosing difficult cases.

\section{CONGENITAL LUNG MALFORMATIONS PRESENTING WITH SIMILAR CHEST X-RAY CHANGES AT BIRTH}

doi:10.1136/archdischild-2012-302724.0615

${ }^{1} \mathrm{G}$ Nepali, $2 \mathrm{M}$ Dattani, 'S Somisetty. 'Neonatal Unit, Luton and Dunstable Hospital NHS Foundation Trust, ${ }^{2}$ Radoiology, Luton \& Dunstable Hospital NHS Foundation Trust, Luton, UK

Congenital defects of diaphragm or malformations of lung usually present in first few hours of birth and if left undiagnosed can lead to significant morbidity and mortality. Early accurate recognition is paramount for subsequent management.

Aim To highlight cases presenting to tertiary neonatal unit with respiratory distress on admission and similar chest x-ray changes.

Case 1 Term infant, one of dichorionic diamniotic twins with antenatal history of polyhydramnios admitted with respiratory distress soon after birth. Initial Chest X-ray (CXR) showing homogeneous opacification of left hemithorax with mediastinal shift to right. Subsequent CT chest revealed Bronchogenic cyst.

Case 2 Term infant admitted with respiratory distress. Initial CXR showed homogeneous opacification of left hemithorax with mediastinal shift to right. Subsequent CXR revealed left sided diaphragmatic hernia.

Case 3 Premature infant born at 34 weeks of gestation with multiple congenital anomalies. Initial CXR showed almost complete opacification of right hemithorax. Chest ultrasound was suggestive of severe right sided diaphragmatic eventration.

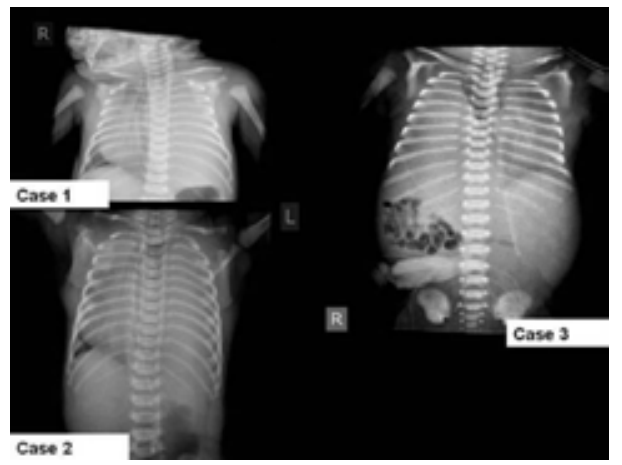

Abstract 615 Figure 1 Initial Chest X-ray 
Conclusion Chest X-ray is a readily available and commonly performed initial radiological investigation in newborns. Although it can detect most common conditions presenting in newborns, it is important to recognize its limitations and consider further imaging as highlighted in our case series.

\section{MORBIDITY DUE TO ACUTE LOWER RESPIRATORY INFECTIONS (ALRI) IN CHILDREN WITH BIRTH DEFECTS: A TOTAL POPULATION LINKED DATA STUDY}

doi:10.1136/archdischild-2012-302724.0616

K Jama-Alol. Centre for Health Services Research, School of Population Health, The University of Western Australia, Perth, WA, Australia

Background Acute lower respiratory infections (ALRIs) remain the leading cause of hospitalisation among children $<2$ years old. Birth defects occur in $5 \%$ of live births in Western Australia (WA), and are a major cause of childhood morbidity and mortality. However ALRI hospitalisation rates among children with various birth defects are unknown.

Methods We conducted a retrospective cohort study of 245,249 singleton births in WA (1996-2005). Population-based hospital morbidity data and the WA Register of Development Anomalies were linked through the Western Australian Data Linkage System to investigate ALRI hospitalisations in children with and without birth defects. We used negative binomial regression to estimate incidence rate ratios (IRR) for the association between birth defects and number of ALRI hospitalisations over the first 2 years of life, adjusting for known risk factors.

Results Overall $11 \%$ of non-Aboriginal children and $40 \%$ of Aboriginal children with birth defects had a least one ALRI admission before age 2 years. In adjusted analyses, Aboriginal and nonAboriginal children were more likely to be hospitalised for ALRI if they had a birth defect than children with no birth defects (IRR 2.29, 95\% CI: 1.89, 2.78; IRR 2.00, 95\% CI: 1.84, 2.17 respectively). Conclusions WA children $<2$ years with birth defects are at greater risk of morbidity due to ALRIs, when compared to children with no birth defects. Risk of ALRI hospital admission varies between different birth defect categories.

\section{FLEXIBLE BRONCHOSCOPY IN THE ACUTE MANAGEMENT OF CONGENITAL LOBAR EMPHYSEMA}

doi:10.1136/archdischild-2012-302724.0617

1.2ES Abd El-Moneim. 'Pediatrics, Taibah University, Madinah Monawara, Saudi Arabia; ${ }^{2}$ Pediatrics, Sohag University, Sohag, Egypt

Introduction Although uncommon, congenital lobar emphysema (CLE) is a potentially life threatening pulmonary abnormality affecting infants. Lobectomy, sometimes done under emergency conditions, is the universally accepted treatment of CLE with severe symptoms. However, in the developing world trained pediatric thoracic surgeons are not everywhere available. On the other hand, the use of flexible fiberoptic bronchoscopy (FFB) in children has shown an excellent safety record. Furthermore, it can be performed under sedation in most cases. We present here a case of CLE, in which we had been able to relieve the acute respiratory distress using FB.

Case presentation and procedure A distressed 4.5 months female infant was referred to us for repetitive prolonged bronchopneumopathy since birth. Successive chest X-rays showed increasing expansion and hyperlucency of right hemithorax, mediastinal shift, and compression of the left lung. At FFB the apicoposterior segmental bronchus of the right upper lobe (APSB/RUL) was narrow, flaccid and showed an expiratory check valve obstruction. By manipulating and rotating the bronchoscope tip into APSB/RUL and applying suction, we had succeeded to release the trapped air in the RUL. Clinical and radiological manifestations resolved completely following the procedure.

Conclusions In addition to the role it might play in the diagnostic workup of patients with CLE, FFB can be used as a tool for relief of obstruction. In certain situations, this may be life saving, especially in places and settings where emergency lobectomy cannot be arranged.

\section{BENEFIT OF PRENATAL DIAGNOSIS OF COMPLEX CONGENITAL HEART DEFECTS ON NEONATAL MORBIDITY}

doi:10.1136/archdischild-2012-302724.0618

'B Ruijsink, ' $\mathrm{F}$ Landsman, ' $\mathrm{M}$ Slieker, ${ }^{2} \mathrm{D}$ Vijlbrief, ${ }^{1} \mathrm{H}$ ter Heide, ${ }^{3} \mathrm{~W}$ de Boode, ${ }^{4} \mathrm{~K}$ Jansen, 'M Freund. 'Paediatric Cardiology; ' ${ }^{2}$ Neonatology, University Medical Center Utrecht, Utrecht; ${ }^{3}$ Neonatology, University Medical Center St. Radboud, Nijmegen; ${ }^{4}$ Paediatric Intensive Care, University Medical Center Utrecht, Utrecht, The Netherlands

Background and Aims As survival rates in neonatal cardiac surgery improve over time, morbidity becomes more important in complex congenital heart disease (cCHD). We investigated whether prenatal diagnosis of cCHD affects preoperative and early postoperative morbidity in neonates.

Methods Retrospective observational study, performed between July $1^{\text {st }} 2004$ and July $1^{\text {st }} 2011$, including 247 infants with cCHD requiring surgery within the neonatal period. 113/247 patients were diagnosed with cCHD by prenatal screening (PreDx). Morbidity was based on preoperative use of high dose prostaglandin $\mathrm{E}$ and preand postoperative cardiac function, ventilation time, length of admission (LOF), inotropic support requirement and presence of severe complications (resuscitation, acute kidney and liver injury, and neurological events)

Results Complexity of cCHD was higher in children diagnosed PreDx. After correction of complexity, median pre-operative ventilation time ( 3 vs 0 days), LOF (7 vs 9 days) and preoperative cardiac function were significantly better in PreDx neonates; and correction of acidosis, preoperative inotropic support and high dose prostaglandin E was significantly less common. Severe complications occurred significantly less when PreDx. There were no significant differences in postoperative morbidity and overall mortality.

Conclusions Prenatal diagnosis of $\mathrm{CCHD}$ improves pre-operative morbidity significantly. The patient condition is better compared to postDx and the need for preoperative intensive care treatment is less. Seemingly, intensive care treatment can achieve a good preoperative condition, even in neonates deteriorated due to an at birth unknown cCHD. This could explain why post-operative morbidity was equal in both groups.

\section{AN OUTBREAK OF NEURAL TUBE DEFECTS IN IRAQ: A CASE REPORT}

doi:10.1136/archdischild-2012-302724.0619

${ }^{1} \mathrm{~S}$ Dastgiri, ${ }^{2} \mathrm{~F}$ Mahmoudzadeh, ${ }^{2} \mathrm{~A}$ Dastgiri. ${ }^{1}$ Community and Family Medicine; ${ }^{2}$ Tabriz University of Medical Sciences, Tabriz, Iran

Aim The aim of this study was to report an outbreak of Neural Tube Defects (NTDs) in Iraq, and to highlight the possible responsibilities of international and local authorities for an action for the control of this outbreak in this region.

Methods Information for the occurrence of NTDs was gathered from reports published from an Iraqi western region, and for other countries from reports published by the International Clearinghouse for Birth Defects and European Network for Surveillance of Congenital Anomalies.

Results Prevalence rate of NTDs was 33 (per 10,000 births, CI95\%: 21-44) in Iraq while different rates have been reported from various parts of the world ranging from 12.6 (per 10,000 births) in Cuba, 9.6 (per 10,000 births) in Norway, 8.7 (per 10,000 births) in China, 7.03 